\title{
Diagnóstico del emprendimiento en Encarnación
}

\author{
Hernán Andrés Schaefer Czeraniuk \\ Hernan.schaefer@unae.edu.py \\ Universidad Autónoma de Encarnación, Paraguay
}

\section{RESUMEN}

La cultura emprendedora forma parte del ADN de la ciudad de Encarnación, reflejada claramente en su actividad comercial. Sin embargo, ante la ausencia de investigaciones que estudien el comportamiento del emprendimiento en la ciudad, este trabajo se propuso diagnosticar el ecosistema emprendedor que lo contiene, a través del estudio analítico de sus integrantes. Esto se ha logrado mediante una revisión bibliográfica de los documentos existentes, entrevistas con expertos y agentes y las encuestas aplicadas a los emprendedores y sujetos con objetivos de emprender.

Esta investigación ha arrojado datos esenciales para el planteamiento y la futura elaboración de políticas públicas de emprendimiento, encontrando factores determinantes dentro del contexto geográfico, las relaciones profesionales, el acceso a la financiación, la actividad en investigación y desarrollo, conflictos en la disponibilidad del capital humano, entre otros.

Palabras clave: cultura emprendedora; emprender; Encarnación 


\title{
Diagnosis of entrepreneurship in Encarnación
}

\begin{abstract}
The entrepreneurial culture is part of the DNA of the city of Encarnación, clearly reflected in its commercial activity. However, in the absence of research that studies the behavior of entrepreneurship in the city, this work aimed to diagnose the entrepreneurial ecosystem that contains it, through the analytical study of its members. This has been achieved through a bibliographic review of existing documents, interviews with experts and agents, and surveys applied to entrepreneurs and subjects with entrepreneurial objectives.

This research has yielded essential data for the planning and future development of public entrepreneurship policies, finding determining factors within the geographical context, professional relationships, access to financing, research and development activity, conflicts in the availability of capital. human, among others.
\end{abstract}

Keywords: entrepreneurial culture; undertake; Encarnacion

Artículo recibido: 02 Setiembre. 2021 Aceptado para publicación: 30 Setiembre. 2021 Correspondencia: Hernan.schaefer@unae.edu.py Conflictos de Interés: Ninguna que declarar 


\section{INTRODUCCIÓN}

Encarnación, con aproximadamente 130.000 habitantes es una de las ciudades más importantes de Paraguay por su contexto cultural (Zuiderwyk, 2018) y por su contexto económico, pues se trata de una de las ciudades de mayor aporte al PIB y de empleo (DGEEC, 2012). Culturalmente, se trata de una sociedad peculiar por la herencia de la plurinacionalidad latente, albergando minorías repartidas entre alemanes, rusos, ucranianos, franceses, japoneses, sirios o libaneses (Otazú, 2011). A esto se le debe sumar la influencia de la cultura misionera de Posadas -Argentina- (Villanueva, 2011), pues Encarnación se encuentra situada estratégicamente por su frontera con dicha ciudad.

Su relación internacional ha sido uno de los puntos más relevantes para el desarrollo de la región con el favorecimiento, entre otros, del comercio fronterizo (Plan Encarnación Más, 2015) (Brites, 2019). Económicamente, su aporte al PIB nacional representa el 3,44\% y un 2,63\% a la remuneración nacional (DGEEC, 2012), hecho incidido también por la devaluación continua del peso frente a la estabilidad del guaraní (Plan Encarnación Más, 2015).

Cabe destacar que la ciudad de Encarnación ha sido epicentro de transformaciones estructurales en los últimos 10 años, resultado de la elevación de la cota del embalse por parte de la Entidad Binacional Yacyretá, ente que gestiona la represa hidroeléctrica de Ayolas, Paraguay (ISEDE, 2008; Brites, 2017; Cañete, 2017). Estas transformaciones trajeron consigo una nueva organización urbanística y nuevas oportunidades comerciales con la creación de una zona exclusiva llamada Circuito Comercial. Esta se encuentra en la cabecera del puente que une Posadas (Argentina) y Encarnación-Paraguay (Schaefer, 2011; Brites, 2019), hecho influido también por el aspecto cultural anteriormente remarcado. A raíz de sus comportamientos semejantes son conocidas como "ciudades gemelas" (Brites, 2019).

En Encarnación, las actividades económicas son casi exclusivas del sector terciario, en donde más del 90\% de los habitantes se dedican al comercio y servicios (DGEEC, 2012), siguiendo la tendencia de lo resaltado por Esteche, Gerhard y Venialgo (2020), cuando aluden a una constitución del ecosistema económico paraguayo de más del $90 \%$ por parte de MIPYMES. Así pues, las actividades de este sector comercio y servicios suponen un $96,5 \%$ de los ingresos de la ciudad y agrupan la mayor cantidad de mano de obra. 
Sin embargo, a pesar de ser una de las ciudades con mayor movimiento económico en Paraguay, Encarnación presenta una desigualdad reflejada en un 33,9\% de pobreza (DGEEC, 2012). Esto muestra que no se trata de emprender por emprender aprovechando transformaciones abruptas o contactos fronterizos cercanos, sino de que las motivaciones principales deben estar vinculadas al desarrollo de una idea y al desarrollo tecnológico (Aulet, 2013). En cambio, un estudio sobre la "Promoción del Emprendimiento y la Innovación Social Juvenil en América Latina (OIT y UNICEF, 2016) mencionaba que el emprendimiento en América Latina es una herramienta para afrontar la falta de oportunidades de empleo, las malas condiciones de trabajo y generar ingresos rápidamente. Dicha tendencia podría estar reproduciéndose en Encarnación, puesto que como señala Kantis (2017) una de las formas más atractivas de acrecentar la empleabilidad de los jóvenes es a través del emprendimiento.

Es importante destacar que el emprendimiento tiene una variada conceptualización, que enfatiza en diferentes aspectos. Shane y Venkataraman (2000) señalan que la mayor parte de la conceptualización se centra en "quién es el emprendedor" y "qué es lo que hace”, pero tiene una limitación cuando debe envolver las oportunidades lucrativas. Así pues, Venkataraman (1997) y Shane y Venkatarama (2000), a pesar de la controversia generada entre los académicos, entienden el emprendimiento como la explicación de "cómo, por quién y con qué efectos" se descubren, evalúan y explotan las oportunidades para crear bienes y servicios futuros. En esta definición se incluye el estudio de las fuentes de oportunidades, el proceso del descubrimiento, la evaluación, la explotación de oportunidades y las personas que precisamente las descubren, evalúan y explotan (Shane y Venkatarama, 2000).

Otras definiciones como las de Carsrud, Brännback y Brännback (2007), lo consideraban como una actitud hacia la gestión; Carrasco y Castaño (2008) lo focalizan en la figura del emprendedor, al cual definen como el fundador de una nueva empresa, un innovador que rompe con la forma tradicional de hacer las cosas y, como lo comprendía Schumpeter (1934), con dotes de liderazgo y talento para identificar el mejor modo de actuar. Vivarelli (2012) describe el emprendimiento como el proceso por el cual se crean nuevas empresas y se vuelven susceptibles de negociación.

En todo esto, también juega un papel importante la forma en que los emprendedores se relacionan con el ambiente, hecho que resulta la manifestación de la cultura emprendedora. 
Esta condicionará los comportamientos y actitudes que asumirán las organizaciones para su desarrollo y relacionamiento con el medio que los rodea (Morales Montejo, 2014). Entran en juego agentes como el Estado, las universidades e, incluso, los mismos emprendedores, donde se genera una comunidad de intercambio de recursos necesarios para el éxito emprendedor que, si se encuentra dispuesta en una zona geográfica, como sería el caso del Circuito Comercial de Encarnación, se le llama "ecosistema emprendedor" (Auletta y Rivera, 2011). Según Urbano y Toledano (2011), las iniciativas son variadas según su naturaleza, la oportunidad de negocios, los recursos disponibles a nivel monetariohumano-material y el conocimiento-habilidades-actitudes de la persona que emprende.

Por eso, este trabajo diagnostica cómo es emprender en Encarnación y cuál es la situación del emprendimiento, ya que como señalan Schnarch (2014) y Kantis (2017), el emprendimiento es un mecanismo para el desarrollo económico y social de las naciones por los cambios en procesos, productos y sistemas que se generan mediante la creación de nuevos puntos de trabajo.

\section{ESTRATEGIAS METODOLÓGICAS O MATERIALES Y MÉTODOS}

Para el diagnóstico de la situación emprendedora en Encarnación se utilizaron la metodología cualitativa y la metodología cuantitativa, describiendo las acciones dentro del ecosistema (Tashakkori y Teddlie, 2003; Gall, Gall y Borg, 2003; McMillan y Schumacher, 2005). No se manipuló ningún tipo de variable, razón por la cual se define como no experimental, mixta y descriptiva.

Para la obtención de datos cualitativos se utilizó una entrevista estructurada, aplicada virtualmente a miembros directivos de distintas entidades, públicas y privadas, que trabajan de manera directa con emprendedores.

Para la obtención de datos cuantitativos, con el fin de comprender el emprendimiento desde la óptica de los emprendedores, describir las características de estos y el proceso en el cual incurrieron para la creación y gestión de sus emprendimientos se aplicó una encuesta creada ad hoc. La muestra de cien $(\mathrm{n}=100)$ emprendedores de un universo difuso por la imposibilidad de cantidad aproximada total y con la incertidumbre de la información/actualización disponible sobre la participación en el ecosistema al momento de la aplicación de la encuesta; fue no probabilística, sujeta a disponibilidad de tiempo y ser responsable legal de, al menos, un emprendimiento. 


\section{RESULTADOS Y DISCUSIÓN}

Para partir hacia el cómo es emprender en Encarnación debemos atender al Plan de Desarrollo Paraguay 2030. En este se plantean estrategias basadas en la transición de la economía dependiente de los recursos naturales hacia la economía del conocimiento.

Los nuevos motores económicos están centrados en el desarrollo con base energética, a través del potenciamiento del ciclo de industrialización vinculado a la utilización de energía eléctrica limpia y renovable, y enfocados en la economía del conocimiento con la diversificación de la economía, con énfasis tecnológico-industrial basado en el aprovechamiento pleno del capital humano. El mismo Plan acomete "la promoción del emprendimiento y el desarrollo empresarial a través del apoyo a empresas nuevas, jóvenes, el estímulo a la creación de fondos de capital privado, incubadoras de empresas, parques industriales y zonas económicas especiales" (Plan de Desarrollo Paraguay 2030, 2014).

Sin embargo, según el ranking "Doing Business 2016" del Banco Mundial, Paraguay se encuentra en el lugar número 135 de 189 entre los países con mayor facilidad para hacer negocios (KOGA, 2016), algo que el Plan Nacional de Desarrollo ya referenciaba al mencionar los principales problemas relacionados a las micro, pequeñas y medianas empresas. A la burocracia del sistema crediticio, se suman la brecha digital, la informalidad, la disponibilidad de capital para equipamiento y un sistema de garantía no operativo. Sobre este, el país cuenta con una Ley de Fondo de Garantía que busca apoyar los emprendimientos de las micro y pequeñas empresas. En el contexto encarnaceno, la ciudad cuenta con múltiples emprendimientos, tal como se referenció anteriormente, puesto que las principales actividades económicas son el comercio y los servicios.

En cambio, el hecho de que Paraguay haya sido víctima de una de las dictaduras más longevas de Latinoamérica repercutió y repercute en que la educación no fuera una insignia del gobierno (Elías y Segovia, 2010). A razón de esto, en Encarnación, la formación superior de los ciudadanos es una actividad reciente, puesto que la universidad más antigua tiene poco más de 60 años y su incursión en ciencia, tecnología e investigación no son áreas de especialidad en la zona (Esteche, 2020).

Como consecuencia de ello, el país está dedicado por excelencia al sector primario, sobre todo en zonas rurales, pero en el entorno urbano de más magnitud social y económica Asunción, Ciudad del Este y Encarnación-, la creación de comercios y servicios es la 
principal forma de vida. ¿Se puede considerar por ello que Encarnación es una ciudad emprendedora o sería preferible considerar que es una ciudad de emprendimientos?

Si se atiende a las definiciones anteriormente tratadas y se trae a colación la definición de Carsrud, Brännback y Brännback (2007), el emprendimiento no es solo crear una empresa, sino es la actitud de vida desde la concepción misma, que incide en la educación, la capacitación, la sostenibilidad, la cooperación y establecimiento de redes, la planificación de la idea de negocio y su ejecución. A continuación, los resultados más destacados:

\section{- Sobre las características personales de quienes emprenden}

Para determinar las características de las personas que emprenden, se realizaron entrevistas a agentes de distintas organizaciones que trabajan directa e indirectamente con el emprendimiento $\mathrm{y}$, al mismo tiempo, se realizaron encuestas a personas con emprendimientos. Al respecto, los emprendimientos son llevados a cabo mayoritariamente por hombres (62\%), con un rango de edad que oscila entre los 24 y los 59 años con un promedio situado entre los 26-32 años.

La mayor parte de las personas encuestadas manifiestan poseer una formación universitaria (82\%), independiente de la carrera estudiada y su relación con el sector al cual se dedica. Sin embargo, el 57\% manifiesta no haber participado en ninguna capacitación sobre el desarrollo de planes de negocios o cultura emprendedora.

\section{- Sobre dónde se emprende}

A partir del diagnóstico realizado por el Ecosistema Urbano en el Plan Encarnación Más (2015), que toma de referencia el Censo Económico realizado en el 2012, más la indagación de documentos disponibles en el archivo de la Municipalidad de Encarnación, los emprendimientos de mayor popularidad son:

1) Comercio al por menor no especializado:

- Hasta el 2012 se registraron 1317 empresas y, desde el periodo 2013 hasta mayo de 2018, se registraron 790 empresas.

2) Comercio al por menor especializado:

- Hasta el 2012 se registraron 1041 empresas y, desde el periodo 2013 hasta mayo de 2018, se registraron 750 nuevas empresas especializadas.

3) Mantenimiento y reparación de vehículos:

- Hasta el 2012 se registraron 370 empresas.

4) Servicios de restaurantes, bares y similares: 
- Hasta el 2012 se registraron 429 empresas y, desde el periodo 2013 hasta mayo de 2018, se registraron 302 nuevas empresas de gastronomía.

5) Actividades de servicios personales:

- Hasta el 2012 se registraban 329 empresas y, desde el periodo de 2013 hasta mayo de 2018, se registraron 95 nuevas empresas de servicios personales.

6) Actividades jurídicas:

- Hasta el 2012 se registraban 229 empresas.

\section{- Financiación del emprendimiento}

La financiación de emprendimientos es objeto de estudio por ser un factor clave para el desarrollo de estos (Megías, 2011). Encarnación tiene los bancos más importantes a nivel nacional, originarios de la ciudad (Palacios 2018), entre otros. Sin embargo, los agentes entrevistados y los emprendedores mencionan con firmeza que el acceso a créditos es una de las principales desventajas al momento de emprender.

Al respecto de los créditos bancarios, los emprendedores se ven en una situación complicada para empresas de nueva creación. En la entrevista al jefe de sección de Marketing del Banco Nacional de Fomento del Paraguay se extrajo que los préstamos sin garantía (llamados a una sola firma) son prácticamente inexistentes para nuevos emprendimientos. Las pocas entidades que ofrecen ese servicio o uno similar ofrecen muy poco capital y disponen de tasas de interés muy elevadas.

Sobre lo disponible estatalmente, de acuerdo con la Ley de la nación número 5628, se creó el fondo de garantía para las micro, pequeñas y medianas empresas, que plantea otorgar garantías y/o re afianzar créditos, operaciones de leasing u otros mecanismos de financiamiento (2016). Se trata de un fondo administrado por la Agencia Financiera de Desarrollo a través del crédito Propymes, sobre el cual los entrevistados opinaron que para acceder a él se requiere de un análisis lento y burocrático, centrado en inversiones a largo plazo, algo contrario a lo que buscan los emprendedores, quienes destacan lo fundamental que es la agilidad.

En cuanto a inversores, los conceptos como "Venture Capital", "Capital Risk" o "Business Angels" están en proceso de desarrollo en la cultura emprendedora encarnacena, por lo que en la actualidad no se piensan en estas vías como fuente de financiación, aunque empiezan a estar disponibles. 
Con todo, las vías de financiación terminan fuertemente vinculadas a la capacidad de ahorro personal, tal como marcaron los entrevistados en un 63,9\%.

\section{- Organizaciones y entidades que intervienen en el desarrollo del emprendimiento} dentro de la ciudad

El estudio parcial del ecosistema emprendedor en Paraguay realizado por KOGA Impact Lab (2016) describe la participación de ciertas entidades en el departamento de Itapúa. Si bien la intervención de estas entidades es visible, no se puede determinar con precisión la constancia con la que desarrollan sus programas. Sin embargo, la mayoría de las organizaciones centran sus actividades exclusivamente en la capital, ya que en el país existe una gran centralización (Nickson, 2017). Esto repercute en que los emprendedores encarnacenos no presentan entusiasmo a la hora de buscar instrucciones en organizaciones especializadas, no han recibido instrucciones sobre la creación de modelos de negocios, ni han realizado actividades de fomento de la cultura emprendedora.

Cabe destacar que existen en el entorno organizaciones sin fines de lucro como ser Ruta Jesuítica, Embajada USA, Universidad Americana, Fenómeno Diseño, Adec, Elevate, Fundación Cird, Incupar, Fundación Paraguaya, F6S y Gen Paraguay (KOGA, 2016). En esta investigación se ha podido comprobar la intervención de Fundación Paraguaya, que implementa soluciones prácticas, innovadoras y sostenibles para la eliminación de la pobreza y la creación de un ambiente digno; también de Incupar, que contribuye a la promoción de las incubadoras de empresas y parques tecnológicos, sensibiliza a actores del sector público y privado, impulsa la investigación, el desarrollo tecnológico y promociona las políticas de apoyo a MIPyMES.

\section{- Universidades y emprendimiento}

Desde lo que suponen las universidades al sistema, tanto por su rol en la formación, la investigación y la transferencia, como por la relación entre empresa-universidad-estado (Sábato, 1970), las universidades pueden trabajar el emprendimiento a través de la divulgación de casos y experiencias, creación de redes, becas de prácticas en empresas, promoción de la cultura innovadora, desarrollo de programas específicos, promoción de la cultura innovadora, etc. (Universia, s.f.).

Actualmente, dentro de las instituciones que trabajan el emprendimiento en la ciudad de Encarnación en alguna o algunas de sus formas se encuentran: 
a) Universidad Nacional de Itapúa: cuenta con una incubadora empresarial, INCUNI, con el objetivo de promover y apoyar la creación y desarrollo de empresas competitivas, proporcionando los servicios necesarios para la generación de ideas mediante el acceso a espacios físicos funcionales, asesoría técnica, asesoría financiera y capacitación.

b) Universidad Autónoma de Encarnación: organiza anualmente el Congreso de Contabilidad Marketing y Empresa donde impulsa el emprendimiento a través del intercambio de experiencias académicas, profesionales e investigativas. Desde el año 2017 desarrolla el concurso UNAE Innovación, competencia de propuestas de modelos de negocios donde el ganador recibe fondos de capital semilla para la apertura de su emprendimiento.

\section{- El gobierno y el emprendimiento}

A nivel regional y municipal, con la Gobernación de Itapúa y la Municipalidad de Encarnación respectivamente, no se visualiza un rol claro ni determinante en el impulso del emprendimiento y la innovación. En la actualidad, no existe una determinada línea de política pública relacionada a la promoción del emprendimiento, hecho que guarda relación con la centralización del país. Los gobiernos regionales no reflejan actualmente una predisposición al trabajo de articulación en el sistema y no se puede hablar de la existencia de instituciones, marcos regulatorios, centros de investigación, apoyo financiero y legislaciones amigables.

En esta línea, los emprendedores encuestados asumen que al momento de emprender no recibirán apoyo del gobierno, hecho que se refleja en que declararon no haber recibido ningún tipo de apoyo de este tipo. Sin embargo, esto no significa la ausencia total del gobierno regional dentro del ecosistema emprendedor, sino más bien la ausencia de una agenda dedicada al fomento e impulso del emprendimiento. Existen trabajos en conjunto realizados con organizaciones para la capacitación de la ciudadanía y personas interesadas en la creación de nuevas empresas, pero no se visualiza ni una política pública ni una constancia para verificar el alcance y la efectividad.

\section{CONCLUSIÓN O CONSIDERACIONES FINALES}

Tras la literatura consultada y el trabajo de campo realizado, se puede afirmar que esta investigación corresponde a la primera incursión específica sobre la descripción del sistema emprendedor de la ciudad de Encarnación. Los antecedentes son muy limitados, lo que 
dificulta la identificación de sujetos, agentes, organizaciones y emprendimientos formalizados.

Los resultados muestran que el ecosistema es incipiente y desorganizado, en donde no se pudo apreciar con claridad los elementos que lo constituyen ni la importancia de los roles de estos dentro de la ciudad. Aunque Encarnación posee una herencia emprendedora y una ciudad de cultura emprendedora, trabajar el emprendimiento desde la copia de modelos exitosos dentro de la ciudad, sumado a la visión de "acceso rápido al mundo laboral", puede suponer un punto negativo ante el cambio de factores externos, tal como ocurre en estos momentos de COVID-19. Por ende, se considera necesaria la evolución de esta tradición emprendedora para reducir la incertidumbre y afrontar las debilidades que la ciudad presenta en cuanto a desigualdad y pobreza, previsiblemente atenuadas en la situación actual.

Cabe destacar que la ciudad presenta ciertas ventajas, como la reducida competencia en rubros específicos, la situación fronteriza que amplía el tamaño de mercado y el hecho de ser la ciudad más visitada del país. Encarnación puede ser uno de los sitios más atractivos para el emprendimiento en el país si se trabaja en la promoción del emprendimiento de manera regulada, articulada e incluida en las agendas públicas.

Esta investigación permite visualizar una serie de lineamientos que pueden ser tenidos en cuenta para el diseño de políticas públicas de emprendimiento para la ciudad, apuntando al desarrollo temprano del ecosistema emprendedor, centrándose en identificar los sectores de mayor productividad y posible mayor productividad, la creación de instituciones para el impulso y regulación del emprendimiento y para el desarrollo de mecanismos de apoyo a este.

\section{LISTA DE REFERENCIAS}

Aulet, B. (2013). Disciplined Entrepreneurship: 24 Steps to a Successful Startup. John Wiley \& Sons.

Auletta, N., \& Rivera, C. (2011). Un ecosistema para emprender. Debates IESA, 12-17.

Brites, W. F. (2015). La ciudad de Encarnación, Paraguay. Intervención urbana a gran escala y nuevos procesos socio-espaciales. Chasqui. Revista Latinoamericana de Comunicación, 33-52. 
Brites, W. F. (2017). Nuevos procesos socio-espaciales en la ciudad de Encarnación, Paraguay: el efecto Yacyretá. En Ciudades, desarrollo y consecuencias sociales de grandes proyectos. Experiencias regionales en análisis. Págs. 213-244.

Brites, W. F. (2019). Ciudades fronterizas, hacia un modelo de desarrollo complementario. La experiencia de Posadas y Encarnación. Memoria del V Encuentro de Investigación en Ciencias Jurídicas Humanas y Sociales "Cuestiones sociales: causas y consecuencias desde el enfoque jurídico, social y educativo". Págs. 27-38.

Cañete, R. (2017). Impactos producidos por la construcción de Yacyreta y el desplazamiento poblacional sobre los niveles de vida de los barrios Arroyo Pora, Sagrada Familia, Pacú Cua y Mboi Ka’e. En Ciudades, desarrollo y consecuencias sociales de grandes proyectos. Experiencias regionales en análisis. Págs. 269-293.

Carrasco, I., \& Castaño, S. (2008). El emprendedor Schumpeteriano y el contexto social. ICE, 121-134.

Carsrud, A., Brännback, M., \& Brännback, M. (2007). Entrepreneurship. Greenwood Publishing Group.

Congreso de la Nación Paraguaya. (2016). LEY N 5628. Fondo de Garantía para las Micro, Pequeñas y Medianas Empresas. Paraguay.

Dirección General de Estadísticas Encuestas y Censos. (2012). Censo Económico. Paraguay.

Elías, R., \& Segovia, E. (2010). La educación en tiempos de Stroessner. En M. d. Culto, La Educación en el Paraguay Independiente. Asunción.

Esteche, E., Gerhard Wasmuth, Y., \& Venialgo Figueredo, M. Las tradiciones organizacionales, culturales y el financiamiento como factores influyentes en las etapas de iniciales y de crecimiento de los emprendimientos en la ciudad de Encarnación. Revista Argentina de Investigación en Negocios, 6(1), 85-96.

Esteche, E. (2020) Diagnóstico de la educación en I+D+I para transferencia de conocimiento en el sector comercial de la ciudad de Encarnación. Tesis de Maestría en Educación y Desarrollo de la Universidad Autónoma de Encarnación. Págs. 7-101.

$\begin{array}{llll}\text { Fundación } & \text { Paraguaya. } & \text { (s.f.). Obtenido }\end{array}$ http://www.fundacionparaguaya.org.py/?lang=es 
Gall, M., Gall, J., \& Borg, W. (2003). Educational Research: An Introduction (7th Edition ed.). Utah: Pearson.

Incupar. (s.f.). Obtenido de http://www.incupar.org.py/

ISEDE, I. S. (2008). Encarnación, ciudad de los siete puentes. Encarnación: Servilibro.

Kantis, H. (2017). La promoción del emprendimiento juvenil. Pensamiento Iberoamericano, 120-130.

KOGA. (2016). Ecosistema Emprendedor en Paraguay. Asunción.

McMillan, J., \& Schumacher, S. (2005). Investigación educativa una introducción. (J. S. Baides, Trad.) Pearson educación.

Megías, J. (7 de Diciembre de 2011). www.javiermegias.com. Obtenido de https://javiermegias.com/blog/2011/12/las-claves-para-crear-un-ecosistemaemprendedor-1/

Morales Montejo, C. (2014). El emprendedor de organizaciones innovadoras. : Siglo del Hombre Editores.

Nickson, R. A. (2017). El lento proceso de la descentralización en Paraguay: el papel del clientelismo. RIEM, 5-24.

Otazú, R. (2011). Encarnación, evolución histórica represada y contemporaneidad. En I. Superior Divina Esperanza, La memoria no se inunda. Encarnación: Servilibro.

Palacios, A. (14 de Junio de 2018). Top 15 entidades financieras en Paraguay con mejores índices. La Nación.

Plan Encarnación Más. (2015). Plan de desarrollo sustentable y plan de ordenamiento urbano y territorial. Ecosistema Urbano, Encarnación.

Programa de las Naciones Unidas para el Desarrollo \& Organización Internacional del Trabajo. (2016). Promoción del emprendimiento y la innovación social juvenil en América Latina. Panamá.

Sábato, J. A., \& Botana, N. R. (1970). La ciencia y la tecnología en el desarrollo futuro de América Latina.

Schaefer, H. (2011). El hombre encarnaceno. En I. S. Esperanza, La memoria no se inunda (págs. 40-41). Encarnación: Servilibro.

Schnarch, A. (2014). Emprendimiento exitoso: Cómo mejorar su proceso y gestión. Colombia: Ecoe Ediciones. 
Schumpeter, J. (1934). The Theory of Economic Development. Cambridge: Harvard University Press.

Secretaria Técnica de Planificación del Desarrollo Económico y Social. (2014). Plan de Desarrollo Paraguay 2030. Asunción.

Shane, S., \& Venkataraman, S. (2000). The promise of entrepreneurship as a field of research. Academy of Management Review, Vol. 25, págs. 217-226.

Tashakkori, A., \& Teddlie, C. (2003). Handbook of Mixed Methods in Social \& Behavioral Research. Thousand Oaks: Sage. 49

Universia. (s.f.). Universia España. Obtenido de Fundación Universia: http://www.universia.es/papel-universidad/desarrollo-profesional/at/1150676

Urbano, D., \& Toledano, N. (2011). Invitación al emprendimiento: Una aproximación a la creación de empresas. Editorial UOC.

Venkataraman, S. (1997). The distinctive domain of entrepreneurship research: an Editor's Perspective. En J. K. Brockhaus, Advances in entrepreneurship, firm emergence, and growth (págs. 119-139). Greenwich: JAI Pess.

Villanueva, L. A. (2011). Identidad del hombre encarnaceno. En I. S. Esperanza, La memoria no se inunda (págs. 36-37). Encarnación: Servilibro.

Vivarelli, M. (Abril de 2012). Entrepreneurship in Advanced and Developing Countries:A Microeconomic Perspective. IZA Discussion Papers(No. 6513).

Zuiderwyk, E. (2018). Encarnación: identidad e interculturalidad. Ed. Divesper. Págs. 7118. 